\title{
Research on the Battery System Technology for Electric Racecar
}

\section{Zhe Cai}

School of Mechanical and Chemical Engineering, University of Western Australia, Perth 6107, Australia

golden_zhe@hotmail.com

Keywords: battery system; electric racecar; safety system control.

\begin{abstract}
In recent years, more and more racecar teams are starting to use electric powertrain systems in their racecars because of the superior torque and power characteristics. The need for a comprehensive study on electric racecar has risen over the last few years. In order to help racecar teams to make informed decisions in component selection and design, this paper covers the knowledge that they need to know about the battery system technology for electric racecars. In this paper, various factors regarding the operation of Lithium Iron Phosphate battery are discussed, providing guidance to build a safe and efficient battery system, also the implementation of related safety system in electric racecars is explained.
\end{abstract}

\section{Introduction}

With a growing concern about pollution and global warming, a flood of demand in electric vehicles from both the public and the government has occurred. In recent years, with more parts and technologies becoming readily available, electric racecars are increasing in popularity due to its competiveness against traditional petrol powered racecars. Compared with ICE(internal combustion engine), electric motors are more versatile and have a low centre of gravity by comparison to ICE. They also have very few moving parts, resulting in less rotational mass, which allows the motors to produce exceptional acceleration response.

In order to provide a better understanding of electric powertrain systems and offer basic guidance on building and testing electric racecars, a detailed study about electric racecars is needed. Battery is an important element in an electric racecar. It allows electrical energy to be harnessed and transform into mechanical energy. The battery cell must be selected carefully in the consideration of various factors, such as weight, cost, and lifespan [1]. Since battery cells are very sensitive to many operational and external conditions, battery management system are needed to monitor and protest battery cell during charging and discharging operations.

This paper includes a comprehensive research on the operation principle of the battery system technology and related safety systems for an electric racecar.

\section{Battery Operation}

Understanding the factors on battery discharging and charging processes is crucial in the battery system design. Some parameters of battery system must be kept in a certain operation range, such as voltage, current, temperature and load.

\section{Battery model}

As is shown in Figure 1, the battery can be model as internal resistance model. This gives:

$$
V_{A}=V_{I}-I \times R_{I}
$$




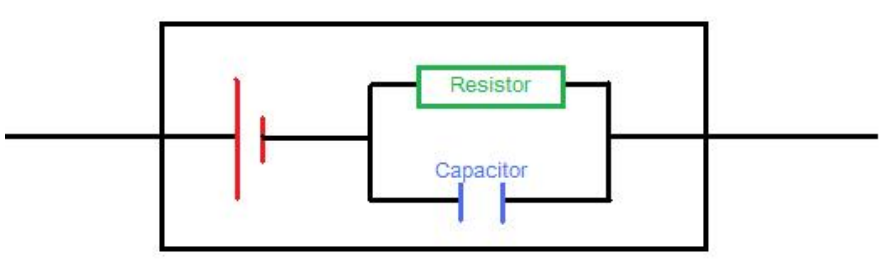

Fig. 1 Internal resistance model

Where $V_{A}$ and $V_{I}$ are the actual and ideal voltage output of the battery, $I$ is the current load, and $R_{I}$ is the internal resistance. Internal resistance is an important factor in battery operation. Internal resistance consumes energy, causing the temperature to increase. The amount of consumed energy is proportional to the load of the battery cell. Internal capacitance stores small amount of energy when the battery cell is not under load, releases it when the battery terminals are connected.

\section{Discharge}

Discharging is the main operation of the battery system. The key determinant of BLDC(brushless direct current) motor control is voltage supplied to motor windings. To fulfill the designed speed and distance range of the motor, the battery system has to be able to supply a stable amount of voltage and capacity during the entire competition. The desired power and torque output are estimated during the motors selection process. As a result, minimum capacity, voltage and current requirements have to be referenced to during the battery design process.

There are four major factors affecting battery discharge performance: SoC(State of charge), cell temperature, discharge rate and aging.

SoC(State of charge): Nominal voltage is only the average voltage output within one discharge process. In practice, voltage output is not constant. Instead, it is proportional to the SoC. Steep voltage drop occurs at about $85 \%$ of the capacity discharged (15\% of SoC) [2]. If the voltage supply is too low, the motor cannot achieve the designed speed inadvertently severely decreasing the performance of racecar. As rule of thumb, $20 \%$ of SoC needs to be left for reserve.

Temperature: The battery temperature is the most important factor on battery performance. Internal resistance of the battery is proportional to temperature. As is shown in Figure 2, the increased internal resistance causes a reduction on actual voltage output of Lithium Ion battery [3]. If the temperature cannot be kept down because of inefficient cooling design, more battery cells will be needed in order to obtain the desirable amount of voltage for the motor to achieve the designed top speed.

Temperature can also affect effective capacity of the battery. For example, according to Figure 2, if designed minimum voltage is 3.6V. At 25 degrees, 3.6V corresponds to $85 \%$ of Depth of Discharge ( $15 \%$ of SoC), but only $65 \%$ of discharge is allowed to maintain minimum voltage at 60 degrees, although Lithium ion batteries have an operational temperature range of -20 to 60 degrees.

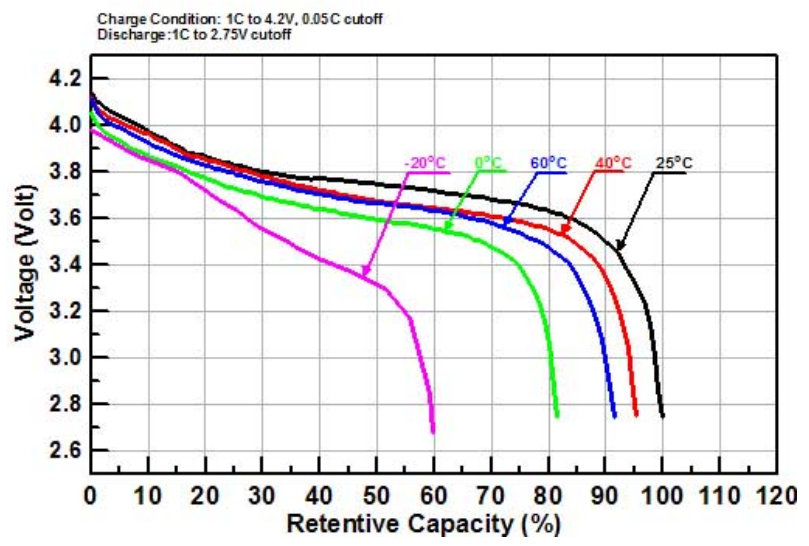

Fig. 2 Temperature effect on voltage and capacity 
Discharge rate: Figure 3 shows the actual voltage of Lithium ion battery under the current load ranging from 1C to 18C [4]. The higher discharge rate (higher current load) causes energy loss due to internal resistance and brings down the actual cell voltage.

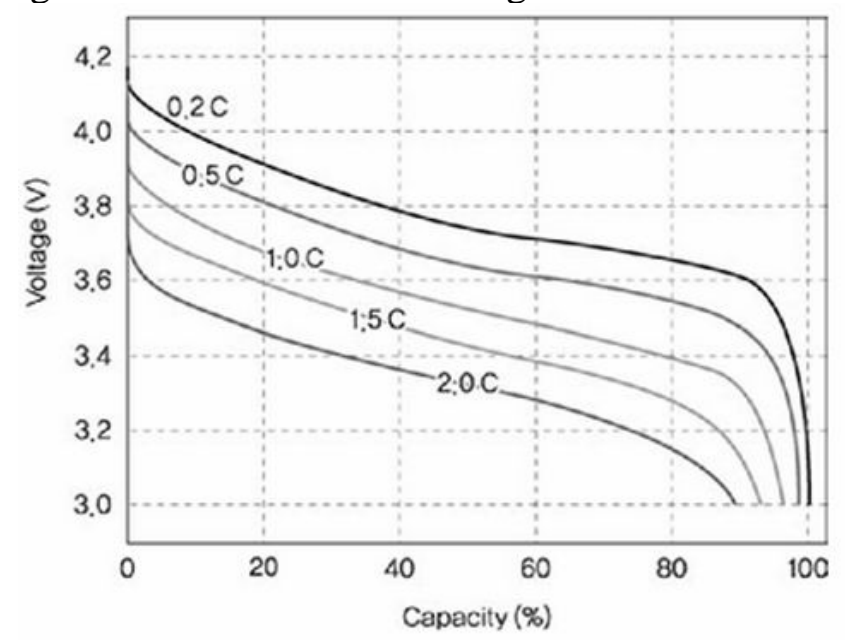

Fig. 3 Cell discharge voltage vs discharge rate

Aging: As the battery goes through charging-discharging cycles, the volume of the active chemicals in the cell is slowly reduced, internal resistance increases, which result in reduced capacity. Figure 4 shows how the battery capacity decreases during its life cycle [4]. The reduction in capacity is not severe, but in practice, the temperature and discharge rate can magnify this trend and cause battery performance issues.

Cycle Life at Room Temperature

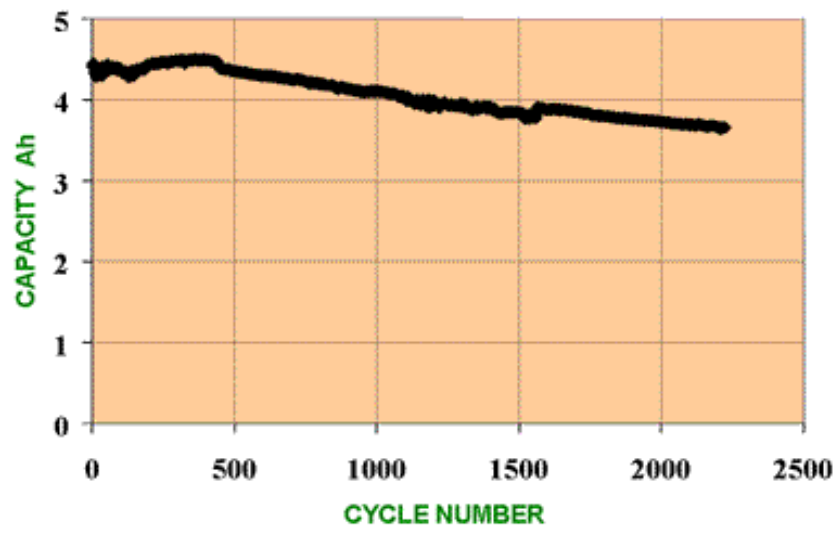

Fig. 4 Capacity of battery vs number of cycle life

\section{Charging}

Charging battery is the reverse process of discharging. There are two factors affecting the charging performance: termination voltage and charging rate.

Overcharging is destructive to Lithium ion battery, because excessive voltages can produce an unwanted reaction which can form layers of insulating materials inside the battery cell. Figure 5 shows how the capacity of LiFePo4 battery cell is dramatically reduced, if charging voltage is higher than $4.2 \mathrm{~V}$ per cell and charging rate is higher than $1 \mathrm{C}$ [5]. As a result, the charging voltage and charging current need to be monitored and controlled carefully. 

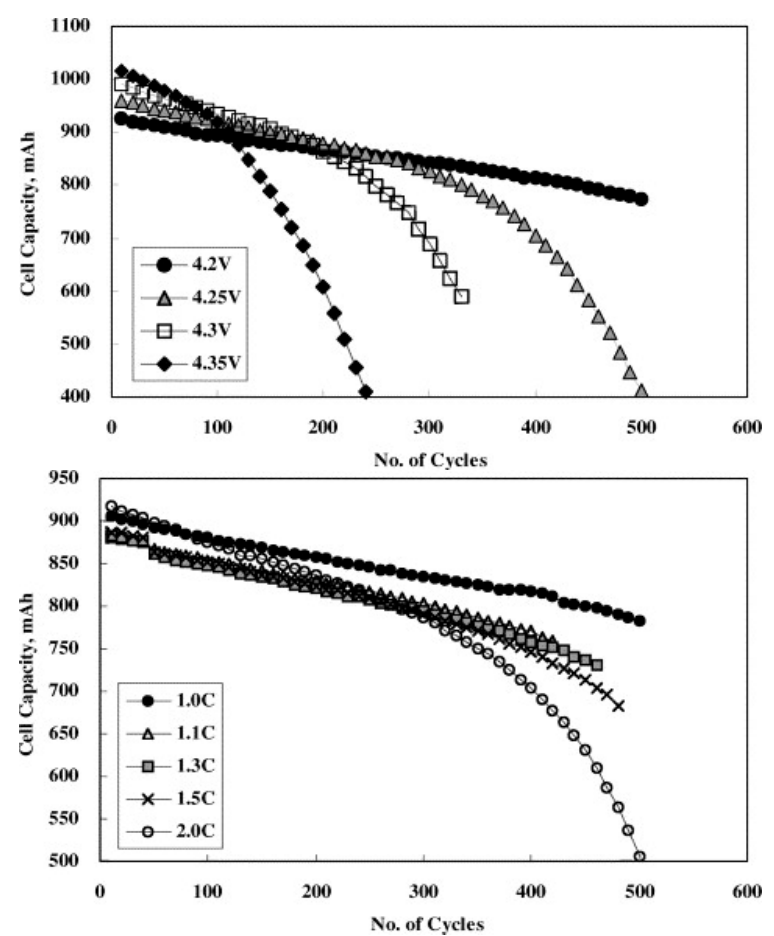

Fig. 5 Effect of overcharging (top) and charging rate (bottom) on the cycle life

\section{Battery Pack}

In order to provide enough voltage and current to the motor controller, a certain number of battery cells have to be accumulated within the battery pack, which is then secured in a battery case.

\section{Accumulation method}

Assuming a battery unit is made of $\mathrm{N}$ identical battery cells connected in series, each battery cell has voltage output of $V_{C}$ and capacity of $Q_{C}$. The voltage output $\left(V_{U}\right)$ and capacity $\left(Q_{U}\right)$ of the battery unit is:

$$
V_{U}=N \times V_{C}, Q_{U}=N \times Q_{C}
$$

$M$ identical battery units are then connected in parallel to accumulate into a battery pack. The accumulated voltage $\left(V_{P}\right)$ and accumulated capacity $\left(Q_{P}\right)$ are:

$$
V_{P}=V_{U}=N \times V_{C}, Q_{P}=M \times Q_{U}=M \times N \times Q_{C}
$$

\section{Battery case}

The function of battery case is to provide a thermal and electrical barrier between the personnel and battery pack and absorb as much force as possible during a crash, so it should be made from strong and insulative material.

\section{Fusing}

The fuse is a passive safety device which disconnects itself if a current passing through exceeds its designed value. The most common over-current situation is short circuit inside battery pack (battery unit/cell insulation fails). Therefore, the fuses are placed in series with all components receiving power from battery pack.

\section{Battery management system}

\section{BMS functions}

BMS(Battery management system) oversees the operation of entire battery pack. The main functions of BMS are:

- Charging management: The battery charger is connected to BMS instead of the battery, therefore BMS can control the current and voltage levels goes into each battery cell during charging, 
and terminate charging when the charging current is below threshold.

- Monitoring: Cell temperatures, current flow and voltage output are all monitored by the BMS in order to protect overstressed cells.

- Cell protection and balancing: As previously mentioned, batteries should not be operated outside its voltage range and temperature limits. In addition, maximum current load should be restricted. The BMS unit can bypass the load/charging current away from weakened and overstressed battery cell, until all cells are in similar conditions.

- SoC estimation: Displaying SoC of the battery pack can help prevent racecar running out of energy during competition.

- Demand management: Power saving mode is achievable by demand management. When power draw from the motor is low or battery pack is running out of energy, the BMS can shut down some of the battery units.

- Safety system control: BMS controls contactors to stop electricity flow out of battery pack. More details will be covered extensively in next section.

\section{Safety system control}

Apart from above-mentioned functions of BMS, BMS must be able to be passively shut down by the safety switches and actively disconnect the motor controller and the battery if there are any potential dangers or error occurs. BMS should be able to shut down the following components:

- Contactors: Contactors are normally-open switches located on both positive and negative sides of the battery pack and receiving power from master BMS to stay closed. If master BMS receives an error signal from BMS units inside the battery pack, such as overheating and short circuit, BMS stops the power supplied to the contactors and force them to be open.

- Torque encoder safety relay: it is similar to contactors, but is located between the motor controller and torque encoder. Without the input from torque encoder, motor controller will stay in standby mode.

- Charger power relay: it is connected in series with battery charger, and it opens when BMS senses charging voltage/current too high or charging process is completed.

- Safety circuit BMS relay: a separate relay is connected in series with the safety switches in case of the contactor failing. Its power supply is independent to the safety circuit.

\section{Construction of BMS}

There are four types of BMS configuration: centralised, master-slave, modular, and distributed.

Distributed BMS is too expensive for a small cell configuration, it is designed for a battery pack with a large individual battery cell. Modular BMS is more suitable for a battery unit with long chain of battery cells in series (large capacity). For FSAE(Formula Society of Automotive Engineering) application, writer recommends the combination of a centralised and master-slave BMS, as shown Figure 6.

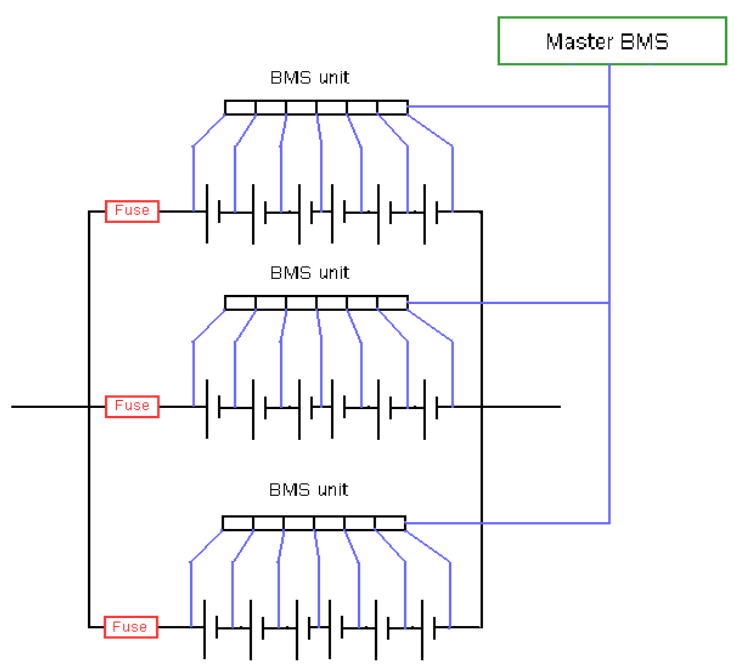

Fig. 6: Centralised master-slave BMS 
In this configuration, there are M BMS units and one master BMS. All BMS units are linked to one daisy chain, which sends data to master BMS. Figure 7 shows that the Daisy chain allows multiple signals to form a pulse like signal.

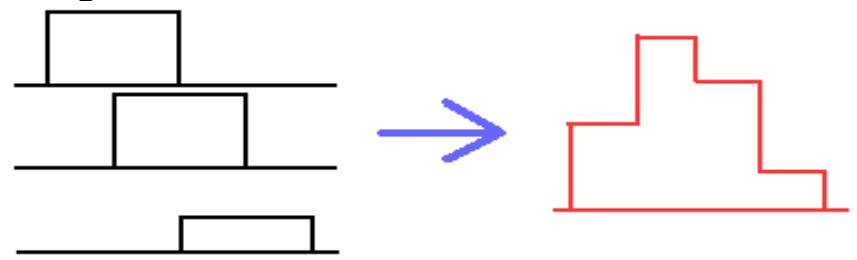

Figure 7: Sample daisy chain signal waveform

\section{Summary}

This paper explores the operation principle of the battery system technology and related safety systems for an electric racecar, and will provide FSAE racecar teams with useful information in decision making and electric racecar building processes in the future. LiFePo4 battery is the battery of choice for FSAE racecars, a large numbers of battery cells are packed into a battery case to form a battery pack, which are monitored and controlled by the BMS. It is highly recommended that Soc should be kept above $20 \%$ and temperature should be kept below $50 \%$ at all the time, and the load on the battery system needs to be secured to protect the battery cells. Other necessary systems of an electric racecar will be discussed in our future work.

\section{References}

[1] Doughty D, Roth E P. A general discussion of Li ion battery safety[J]. Electrochemical Society Interface, 2012, 21(2): 37-44.

[2] Principles and applications of lithium secondary batteries[M]. John Wiley \& Sons, 2012.

[3] Choi S S, Lim H S. Factors that affect cycle-life and possible degradation mechanisms of a Li-ion cell based on LiCoO 2[J]. Journal of Power Sources, 2002, 111(1): 130-136.

[4] Moura S J, Chaturvedi N A, Krstic M. PDE estimation techniques for advanced battery management systems_-Part I: SOC estimation[C]//American Control Conference (ACC), 2012. IEEE, 2012: 559-565.

[5] Andrea D. Battery Management Systems for Large Lithium Ion Battery Packs[M]. Artech House, 2010. 